Catinca Adriana Stan

Université Laval

\title{
Delâge, D. et Warren, J.-P. (2017). Le piège de la liberté: les peuples autochtones dans l'engrenage des régimes coloniaux. Montréal, QC : Boréal.
}

\section{RECENSION}

La question autochtone surgit dans l'actualité avec un élan sans précédent : plus de détails font surface - comme le drame des enfants des pensionnats, la violence envers des femmes, les ravages de l'alcool et de la drogue, la pauvreté et la misère de certaines réserves -, plus on se sent dépourvu, divisé, impuissant en tant que société qui est appelée à agir, à faire sa part pour qu'une véritable réconciliation puisse avoir lieu.

Parmi les nombreux écrits qui traitent des enjeux autochtones, le livre Le piège de la liberté: les peuples autochtones dans l'engrenage des régimes coloniaux (Montréal, Boréal, 2017), rédigé par deux sociologues de renommée internationale, Denys Delâge, de l'Université Laval, et Jean-Philippe Warren, de l'Université McGill, s'impose comme un incontournable. En effet, par la profondeur de leurs propos, par la rigueur du travail documentaire, par le respect et la sensibilité dont ils font preuve tout au long de leur récit envers les communautés autochtones, les auteurs construisent un discours équilibré, convaincant, qui aide le lecteur à saisir les racines profondément ancrées dans l'histoire coloniale des problèmes autochtones d'aujourd'hui.

Structuré en sept chapitres, le livre explique le processus d'acculturation des Autochtones, au nom des croyances et des idéologies occidentales. Le premier chapitre trace les grandes lignes de l'organisation sociale des peuples autochtones. On voit entre autres que l'exercice du pouvoir s'exerce par des chefs élus dont l'autorité est limitée à la parole, le système de don et de contre-don qui exige une réciprocité entre les individus, les 
plaçant en constante dette envers les autres membres, etc. Le deuxième chapitre met l'accent sur les " rapports inégaux et combinés » (Novack, 2009) institués dès le début de la traite de fourrures. Ainsi, on comprend qu'au désavantage technologique on ajoute celui de l'écriture, signe de la vérité figée et incontestable, par rapport à la vérité vivante de la parole. Il s'en suit dès lors une décrédibilisation des sources orales, du récit autochtone relatant la dette contractée par les premiers Euro-Canadiens envers les populations les ayant accueillis et ayant partagé leurs terres.

Le troisième chapitre explique les rapports coloniaux selon une triple conquête du monde, à la fois par une religion propagandiste (idée qu'on retrouve également dans un autre livre de Delâge, Lepays renversé, où l'on présente les privilèges des convertis, notamment un meilleur prix pour les fourrures et le droit de posséder des armes à feu), une politique impérialiste d'expansion selon le principe de terra nullius et un système capitaliste dont le pouvoir est fondé sur la coercition. Une série de trois autres chapitres exposent les principes et les valeurs libérales du régime anglais (1760-1867) : le commerce, le travail régulier et la propriété privée. Ce faisant, les auteurs démontrent l'ampleur du choc entre des cultures très différentes et la lente marche vers ce que les Euro-Canadiens considéraient comme le progrès et les Autochtones comme une aliénation et un désaveu de leur propre culture. On voit notamment dans ces trois chapitres la différence entre l'idéologie du Régime français et celle du Régime britannique à l'égard des Autochtones. Contrairement à la société française dans laquelle la soumission à l'autorité suffisait à l'intégration au régime (les «Sauvages » pouvant rester eux-mêmes), la société britannique, basée sur l'idéologie libérale, exigeait de la part des membres l'intériorisation d'un corpus de valeurs essentielles, notamment le travail de la terre. Car, selon cette idéologie, l'homme moderne domine la nature et doit la mettre à sa main : "Le signe distinctif de la civilisation consistait à défricher et à ensemencer les terres» (p. 344). Cela signifie que le travail de la terre devient le critère par excellence qui départage ce qui est « civilisé » et ce qui ne l'est pas. Le refus ou l'incompréhension de ces valeurs libérales allait maintenir les Autochtones dans un état marginalisé et légitimer l'oppression envers eux.

Le dernier chapitre traite de la douloureuse question des pensionnats autochtones.Placés en dépendance dès le XIX ${ }^{\mathrm{e}}$ siècle, ils étaient régis par la Loi sur les Indiens (1876) qui encourageait l'émancipation individuelle, afin de diminuer le poids de la dette historique. Pour «civiliser les Sauvages", le gouvernement fédéral a mis en place des politiques assimilatrices, en même temps qu'il a créé les réserves. Il a amené entre autres la loi du sang, externe aux peuples concernés, qui décide dorénavant qui est Indien et qui ne l'est pas. Véritable « génocide culturel » (p. 401), le pensionnat connaît un échec, alors expliqué par l'incapacité des Autochtones de vivre et d'apprécier la liberté. Laissés à eux-mêmes, les Autochtones s'entêtent, comme jadis les Canadiens français, à ne pas disparaître " naturellement ».

\section{La pertinence de ce livre en éducation}

L'histoire enseignée à l'école insiste sur les sociétés autochtones avant l'arrivée européenne, tout en gardant le silence quant au rôle des Autochtones entre le XVII ${ }^{\mathrm{e}}$ et le $\mathrm{XX}^{\mathrm{e}}$ siècle (Stan, 2015), soit les siècles durant lesquels les pratiques de dépossession culturelle et les structures expliquant les conditions sociales actuelles furent mises en place. Ce livre constitue alors une ressource importante pour combler le vide historiographique, notamment parce qu'il explique comment s'est fait le passage d'une société qu'on considère au $\mathrm{XVII}^{\mathrm{e}}$ siècle comme étant libre et la marginalisation qui s'accentue à partir du 
$\mathrm{XIX}^{\mathrm{e}}$ siècle. Pour tout enseignant qui traite de l'histoire du Québec, il est impératif de comprendre ce changement de paradigme, puisque faire l'histoire de la société québécoise, c'est faire l'histoire de tous ceux qui l'ont construite.

Par le contraste qu'offre le mode de vie autochtone, ce livre projette un regard critique envers la société occidentale, permettant de s'interroger sur les fondements mêmes d'un système qui avantage les détenteurs de capital. Dans ce sens, il est pertinent pour tous ceux qui veulent comprendre davantage la société actuelle, notamment les chercheurs en éducation, puisque la pertinence de l'acte éducatif par rapport à un public socialement et culturellement hétérogène demeure une question centrale.

\section{Références}

Delâge, D. (1991). Le pays renversé : Amérindiens et Européens en Amérique du Nord-Est, 1600-1664. Montréal, QC : Boréal.

Novack, G. (2009). Understanding history: Marxist essays. New York, NY : Pathfinder.

Stan, C. A. (2015). De peuple sauvage à peuple fondateur : l'image des Amérindiens et des Daces dans les manuels scolaires du Québec et de la Roumanie. Acta iassyensia comparationis, 15(1), 234-246. Repéré à

http://www.literaturacomparata.ro/Site Acta/Old/acta15/27.AIC 15 Stan.pdf

\section{Pour citer cet article}

Stan, C. A. et Vallée-Longpré, J. (2018) Delâge, D. et Warren, J.-P. (2017). Le piège de la liberté : les peuples autochtones dans l'engrenage des régimes coloniaux. Montréal, QC : Boréal. Formation et profession, 26(3), 134-136.

http://dx.doi.org/10.18162/fp.2018.157 